\title{
SELECTING VERIFICATION AND VALIDATION TECHNIQUES FOR SIMULATION PROJECTS: A PLANNING AND TAILORING STRATEGY
}

\author{
Zhongshi Wang \\ ITIS GmbH \\ Werner-Heisenberg-Weg 39 \\ D-85577 Neubiberg, GERMANY
}

\begin{abstract}
Conducting verification and validation $(\mathrm{V} \& \mathrm{~V})$ of modeling and simulation $(\mathrm{M} \& \mathrm{~S})$ requires systematic and structured application of different $V \& V$ techniques throughout the $M \& S$ life cycle. Whether an existing technique is appropriate to a particular $\mathrm{V} \& \mathrm{~V}$ activity depends not only on the characteristics of the technique but also on the situation where it will be applied. Although there already exit several guidance documents describing a variety of $\mathrm{V} \& \mathrm{~V}$ techniques and their application potential, accessible findings or experiences on the effective selection of suitable $\mathrm{V} \& \mathrm{~V}$ techniques for a given $M \& S$ context are still lacking. This paper presents: 1.) a characterization approach to developing a $\mathrm{V} \& \mathrm{~V}$ techniques catalog that packages the available techniques together with the information about their application conditions; and 2.) a planning and tailoring strategy for project-specific selection of the appropriate V\&V techniques from the established catalog according to the goals and characteristics of a simulation study.
\end{abstract}

\section{INTRODUCTION}

For reasons of increasing productivity, efficiency, and decreasing time and costs for system innovations, modeling and simulation (M\&S) becomes a standard "tool" for a huge variety of applications. As this trend will be an ongoing one, the demand for controlling and demonstrating the quality of a simulation model and its application is obvious. Verification and validation $(\mathrm{V} \& \mathrm{~V})$ focuses on assessing the accuracy quality characteristic of an M\&S application with respect to its objectives (Balci 1998b, Shannon 1975), and is intended to ensure that only correct and suitable models and simulation results are used in application practice.

Conducting model verification and validation requires systematic selection and application of different $\mathrm{V} \& \mathrm{~V}$ techniques throughout the $\mathrm{M} \& \mathrm{~S}$ life cycle. Although a wide variety of $\mathrm{V} \& \mathrm{~V}$ techniques (more than 100) are commonly available, only a limited number of techniques are considered in practice (Balci et al. 2002). According to Vegas and Basili (2005), the main reasons for this deficit are: 1.) theoretical information about techniques is normally distributed across different sources; and 2.) empirical knowledge about techniques application is generally not accessible. Thus, due to lack of information the selection of $\mathrm{V} \& \mathrm{~V}$ techniques heavily relies upon the personnel experience of a $\mathrm{V} \& \mathrm{~V}$ agent and is conducted typically in an ad hoc manner.

Regarding selection and application of $\mathrm{V} \& \mathrm{~V}$ techniques in an $\mathrm{M} \& \mathrm{~S}$ project, several approaches have been proposed. Deslandres and Pierreval (1991) present a knowledge-based approach to automatic selection and application of an appropriate statistical V\&V technique for certain model behavior validation, in which statistical tests are used to analyze the simulation model output behavior in comparison to the output behavior measured from the real system or estimated by experts. The objective of this approach was originally intended 1.) to regroup the existing $V \& V$ techniques (e.g., in the literature) and represent them in the validation expert system, then 2.) to extend the the expert system by integrating additional 
experts knowledge on statistics, modeling and simulation, and V\&V. However, due to the difficulty in designing such a validation expert system, severe restrictions and limitations had to be made for developing a prototype: 1.) the investigated system must be observable. This implies that it is possible to collect data on the operational behavior of the system (Sargent 2005); 2.) the V\&V techniques are limited to statistical tests comparing real system data and simulated data; and 3.) only stochastic and discrete event models (particularly manufacturing systems models) are considered. In addition, the application of this approach and its further development are not sufficiently reported.

Concerning the application of V\&V techniques for general use, Balci (1998b) investigates approximately eighty techniques applicable to conducting model $\mathrm{V} \& \mathrm{~V}$ and proposes a taxonomy that categorizes the available V\&V techniques in four groups: informal, static, dynamic, and formal, describes their characteristics, and outlines their possible applicability in the M\&S life cycle. With an illustrative example, Balci (1998a) shows the general application potential of the available techniques in the different $\mathrm{V} \& \mathrm{~V}$ stages for two M\&S life cycle processes. Based on Balci's taxonomy, the DoD VV\&A Recommended Practices Guide for V\&V techniques (Department of Defense 2001) is proposed. Although Balci's taxonomy summarizes and specifies the majority of $\mathrm{V} \& \mathrm{~V}$ techniques applicable in the $\mathrm{M} \& \mathrm{~S}$ context and can even serve as a reference work for application of the $\mathrm{V} \& \mathrm{~V}$ techniques, it alone is still insufficient for conducting techniques selection. That's because whether a V\&V technique should be selected for a particular V\&V activity depends not only on the characteristics of the technique but also on the context of its application. The way of project-specific techniques selection is however not sufficiently discussed in these approaches.

This paper introduces an M\&S-specific characterization scheme including relevant attributes to identify and specify the information required for selecting $\mathrm{V} \& \mathrm{~V}$ techniques. Considering the $\mathrm{V} \& \mathrm{~V}$ techniques summarized in Balci's taxonomy, an organization-specific techniques catalog can be established by using the defined characterization scheme. Such a V\&V techniques catalog works as an information repository supporting the techniques selection. The proposed characterization approach can be applied to any simulation study with well defined and structured model development and $\mathrm{V} \& \mathrm{~V}$ process.

The purpose of this paper is fourfold: 1.) to present the characterization approach applied in this work; 2.) to discuss the attributes defined the characterization scheme; 3 .) to illustrate how this scheme can be used to establish a $\mathrm{V} \& \mathrm{~V}$ techniques catalog; and 4.) to demonstrate the project-specific selection of the appropriate $\mathrm{V} \& \mathrm{~V}$ techniques from the $\mathrm{V} \& \mathrm{~V}$ catalog for a simulation study.

\section{A CHARACTERIZATION APPROACH TO SELECTING V\&V TECHNIQUES}

This section gives an overview of the characterization approach applied in this work and discusses some relevant issues to be considered, when constructing an M\&S-specific characterization scheme to specify the properties of $\mathrm{V} \& \mathrm{~V}$ techniques.

\subsection{Characterization of $\mathrm{V} \& \mathrm{~V}$ Techniques}

In the software community, Vegas, Juristo, and Basili (2003) propose a characterization scheme to support the selection of software testing techniques. For gathering the relevant information about software testing techniques, the proposed scheme defines a list of parameters, which are hierarchically arranged in three categories: Level, Element, and Attribute. Three information levels (tactical, operational, and historical) are defined in the scheme. Each level consists of several elements, which in turn are subdivided into attributes. For example, the tactical level includes two elements: Objective and Scope. The tactical element Objective consists of attributes such as: purpose of the testing technique, defect types the technique helps to detect, and effectiveness of the technique.

For the purpose of instantiation, an existing testing technique can be characterized according to the attributes defined in the scheme. For example, the technique boundary value analysis has the purpose of finding defects, the defect type it helps to defect is control, its effectiveness is that it can find $50 \%$ of 
control defects, the comprehensibility of its application is high, and the application cost is low, etc (Vegas 2002). Once a set of the available techniques are characterized, a techniques repository is build.

Despite its applicability to selection of test case strategies for software projects, this characterization scheme however cannot be used for selecting V\&V techniques in the M\&S context, because:

- this approach is only applicable to the testing phase of the software development life cycle, model $\mathrm{V} \& \mathrm{~V}$, however, should be conducted as a continuous activity throughout the M\&S life cycle;

- this scheme is designed specifically for characterizing testing case strategies, other types of techniques like inspections, reviews, and walkthroughs are hard to characterize;

- the attributes in the scheme are defined for software projects and are not always applicable to a simulation study;

- the selection of more than one testing technique is not possible for a given (software) project (Dias-Neto and Travassos 2009);

- no process is associated with the scheme to support the techniques selection (Wojcicki and Strooper 2007).

Thus, an improved scheme needs to be developed for characterizing $\mathrm{V} \& \mathrm{~V}$ techniques. In contrast to the concept described in Vegas and Basili (2005), an M\&S-specific characterization is established. In addition, an explicit step for techniques selection is introduced.

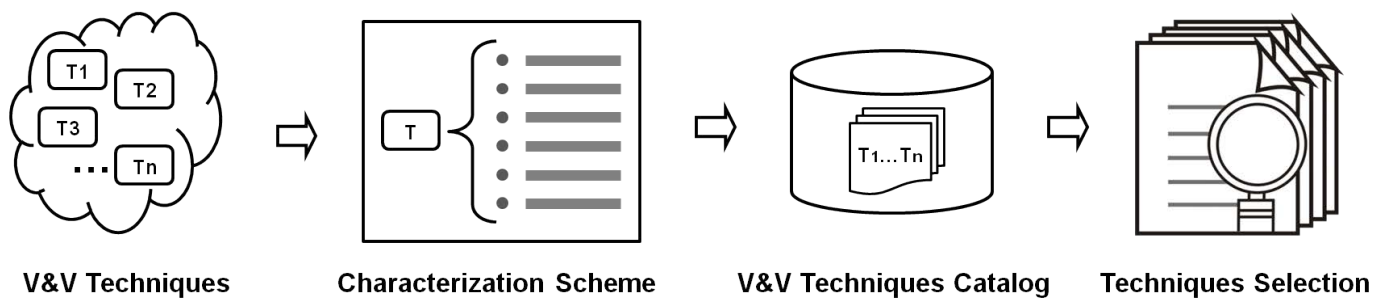

Figure 1: Characterization approach in this work

As illustrated in Figure 1, the characterization approach proposed in this work can be outlined as follows:

1. Analyzing the available $\mathrm{V} \& \mathrm{~V}$ techniques applicable to $\mathrm{M} \& \mathrm{~S}$ applications;

2. Defining an M\&S-specific characterization scheme specifying the relevant properties of $\mathrm{V} \& \mathrm{~V}$ techniques;

3. Using the defined scheme to characterize the existing $V \& V$ techniques and building a catalog of the characterized $\mathrm{V} \& \mathrm{~V}$ techniques;

4. Selecting the appropriate $V \& V$ techniques from the $V \& V$ techniques catalog according to the goals and characteristics of an $M \& S$ project.

\subsection{Issues Relevant for Developing an M\&S-Specific Characterization Scheme}

\subsubsection{Model Deficiencies}

In the software community, despite some different terminologies, the relationship between the cause of a software defect and its consequences is consistently specified. In general, as defined in an IEEE Standard (IEEE 1990), an error is a human oversight or wrong decision, which results in a fault, or a defect within the software (including requirements specifications, design, and code). When the software is executed, a fault, or a combination of faults, may (or may never) cause a failure. In many cases, the term "defect" is used in a generic manner referring to a fault, a failure, or even an error. Compared to a software defect, the meaning of what is wrong or inaccurate in a model appears more complex, just as the well-known 
statistician George Box stated: "All models are wrong, some are useful". From the V\&V viewpoint, two sources of $M \& S$ deficiencies can be identified within the $M \& S$ life cycle. On the one hand, since $M \& S$ requires intensive investment of human efforts, and includes software development, the causal structure of human errors, faults, and failures also exists in simulation models.

On the other hand, due to lack of knowledge, e.g., uncertainty (Oberkampf et al. 2002) in the different M\&S development phases, or for the purpose of model simplification, assumptions and approximations must be made. Whether they are accurate enough or actually acceptable can only be estimated with respect to the specified objectives of the model by particular V\&V techniques such as face validation (Balci 1998b, Shannon 1998, Law and Kelton 2007). Thus, inaccuracies are those deficiencies in the model, which result from improper or unacceptable assumptions and approximations made.

In some cases, although the individual assumptions and approximations are estimated as acceptable, the effect of their aggregation could still cause unacceptable inaccuracies. In the course of M\&S development, improper assumptions and approximations cause further inaccuracies which can eventually lead to insufficient model credibility, i.e., the model is not useful. Thus, both sources of M\&S deficiencies must be handled by the V\&V activities.

\subsubsection{V\&V Techniques: Subjective vs. Objective}

The nature of $M \& S$ deficiencies motivates the application of both subjective and objective $V \& V$ techniques. Subjective techniques rely heavily on individuals' insights and intuitions, particularly opinions of subject matter experts (SMEs), e.g., face validation or Turing tests. Objective techniques on the contrary are based primarily on statistical methods or mathematical procedure, such as hypothesis tests or confidence intervals (Sargent 2005).

Compared to objective methods, subjective techniques are used in a readily comprehensible manner, and can be regarded as the only possibility, when no objective methods are actually applicable. However, estimates of SMEs are not always reliable (Overstreet 2002), or experts may differ in their opinions about the model estimation. On the other hand, objective techniques can provide the unique experimental evidence, but their application is usually presupposed by specific conditions and requires the mastery of particular knowledge or skills for testing personnel (V\&V agents). Therefore, whether an objective or a subjective technique should be selected for a particular V\&V activity is basically situation dependent. In general, a combination of them are to be used. Walton, Patton, and Parsons (2001) discuss the application of the two technique classes in the context of military simulations.

\subsubsection{Structured Model V\&V}

Balci (1994) outlines several relevant differences between M\&S applications and general software engineering: 1.) simulation modeling is an art (Shannon 1975) and should be integrated in a simulation study; 2.) the simulation results are obtained with experimenting the developed simulation model. This requires an additional process for planning, designing model experiments, and executing simulation runs; and 3.) the results are descriptive and have to be analyzed and interpreted. These identified differences indicate that a simulation study is conducted in a more complex context than software development and model $\mathrm{V} \& \mathrm{~V}$ (including $\mathrm{V} \& \mathrm{~V}$ techniques selection) should be performed in a structured way.

Figure 2 illustrates a general description of structured M\&S and V\&V (Wang and Lehmann 2010). From the development's point of view, a simulation study is initiated by presenting sponsor needs and consists of a set of ordered development phases. In each phase a defined work product is developed such as $M \& S$ requirements specification, conceptual model, formal model specification, and executable model. A work product consists of several content-related subjects, each of which represents a specific issue or a model element. From the V\&V point of view, each model development activity is coupled with a corresponding $V \& V$ effort throughout the $M \& S$ life cycle. Consequently, three kinds of parties are involved in model development, $\mathrm{V} \& \mathrm{~V}$, and project management, i.e., participants from the sponsor 


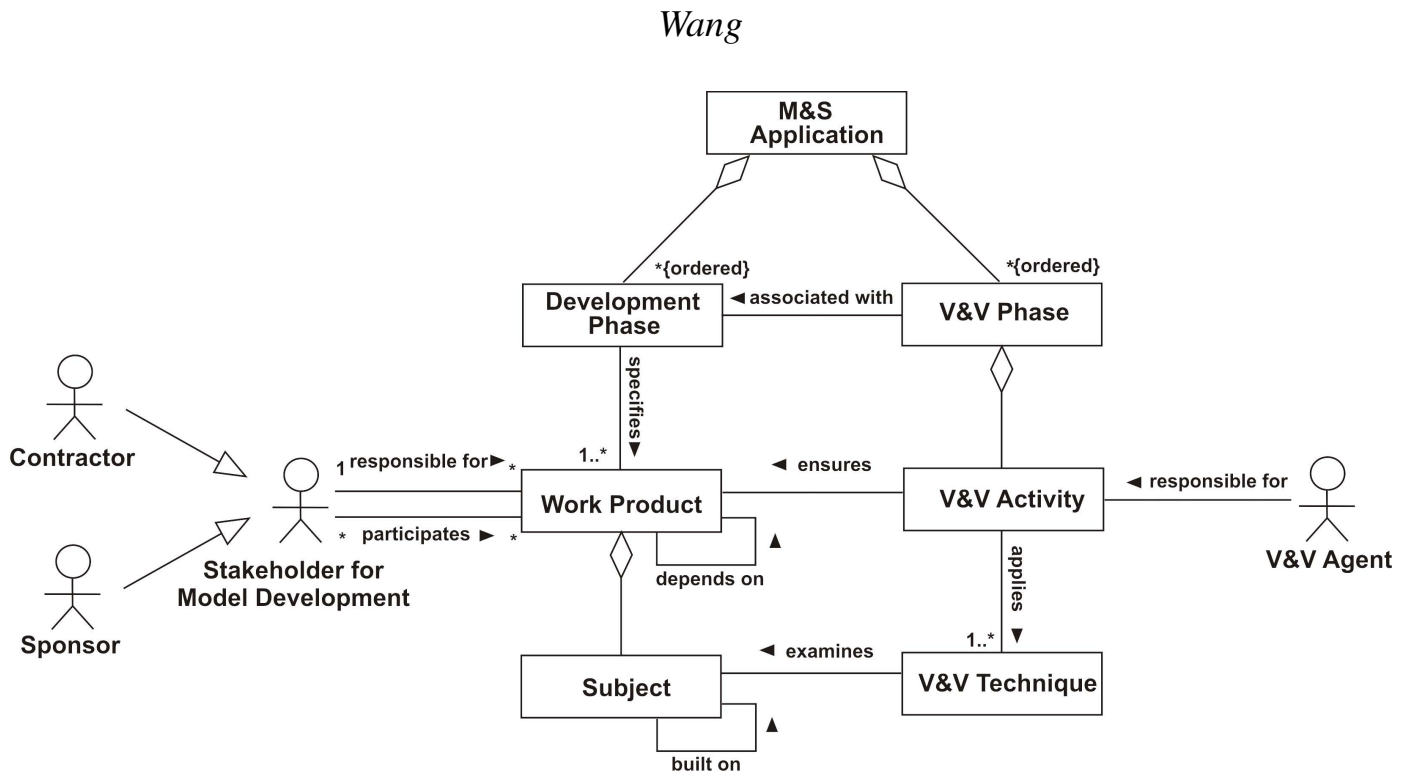

Figure 2: Structured M\&S and V\&V

side, the developer side, and the V\&V institution (Balci, Nance, Arthur, and Ormsby 2002). Since model $\mathrm{V} \& \mathrm{~V}$ should be conducted in an independent manner, the application of independent V\&V (IV\&V) is an emphasis of this paper. Arthur and Nance (2000) discuss the benefits of IV\&V for a simulation study.

\section{M\&S-SPECIFIC CHARACTERIZATION SCHEME}

Since model V\&V is a continuous activity throughout the entire $M \& S$ life cycle, the characterization approach should facilitate systematic technique selection. Hence the objectives of the proposed characterization scheme can be outlined as follows:

- the characterization scheme should provide support to select appropriate techniques for each V\&V activity throughout the M\&S life cycle;

- information aiding the project management with planning the V\&V effort should be included;

- the proposed characterization should be applicable to any simulation study with well defined and structured model development and $\mathrm{V} \& \mathrm{~V}$ process.

Selection of $\mathrm{V} \& \mathrm{~V}$ techniques is a decision process to determine, whether or not application of a technique and costs of its application match a given $M \& S$ context. Therefore, when constructing an M\&S-specific characterization, two issues, i.e., the applicability of techniques and the costs of their usage, are crucial and have to be included in form of several suitable attributes. Based on this consideration, the attributes defined in the characterization scheme are organized in two categories: Applicability and Cost.

The category Applicability includes attributes to indicate:

- the application domain of a V\&V technique within the $M \& S$ life cycle, i.e., the information about in which $\mathrm{V} \& \mathrm{~V}$ activities, to which artifacts of a model a technique can be applied, and which type of model deficiencies it helps to detect.

- the operational conditions, i.e., whether application of a V\&V technique is coupled with a particular development paradigm of a simulation study, modeling formalism, simulation type, and simulation language; or whether a observable system (in which it is possible to collect data on the operational behavior) or execution of a model is required.

- the way of technique application, e.g., a V\&V technique is subjective or objective, whether its application is dependent on application of other techniques. 
Table 1: Characterization scheme description

\begin{tabular}{|c|c|c|}
\hline Category & Attribute & Description \\
\hline \multirow[t]{11}{*}{ Applicability } & V\&V activity & $\begin{array}{l}\mathrm{V} \& \mathrm{~V} \text { stages in which the } \mathrm{V} \& \mathrm{~V} \text { technique can be applied, } \\
\text { such as } \mathrm{V} \& \mathrm{~V} \text { of } \mathrm{M} \& \mathrm{~S} \text { requirements specification, } \mathrm{V} \& \mathrm{~V} \\
\text { of conceptual model, } \mathrm{V} \& \mathrm{~V} \text { of executable model, } \mathrm{V} \& \mathrm{~V} \text { of } \\
\text { data, etc. }\end{array}$ \\
\hline & Object & $\begin{array}{l}\text { Artifacts that the } \mathrm{V} \& \mathrm{~V} \text { technique is able to examine, in- } \\
\text { cluding work products, their documentation, and any other } \\
\text { documents created in the course of a simulation project. }\end{array}$ \\
\hline & Deficiency type & $\begin{array}{l}\text { Types of model deficiencies that the technique helps to } \\
\text { detect. }\end{array}$ \\
\hline & Development paradigm & $\begin{array}{l}\text { Model development paradigm to which the technique is } \\
\text { linked, e.g., component-based, object-oriented, agile de- } \\
\text { velopment, procedural paradigm, etc. }\end{array}$ \\
\hline & Modeling formalism & $\begin{array}{l}\text { Modeling formalism to which the technique is linked, e.g., } \\
\text { Petri nets, process algebra, Discrete Event System Speci- } \\
\text { fication (DEVS) (Zeigler, Praehofer, and Kim 2000), etc. }\end{array}$ \\
\hline & Simulation type & $\begin{array}{l}\text { Simulation types to which the technique is linked, e.g., } \\
\text { stochastic, deterministic, discrete or continuous event sim- } \\
\text { ulation. }\end{array}$ \\
\hline & Simulation language & $\begin{array}{l}\text { Simulation language to which the technique is linked, in- } \\
\text { cluding general-purpose programming languages, general- } \\
\text { purpose simulation language, and special purpose simula- } \\
\text { tion packages. }\end{array}$ \\
\hline & Observable system & $\begin{array}{l}\text { Whether or not an observable system is required for ap- } \\
\text { plying the technique? }\end{array}$ \\
\hline & Model execution & $\begin{array}{l}\text { Whether model execution is required, when applying the } \\
\text { technique. }\end{array}$ \\
\hline & Modality & Is the technique subjective or objective? \\
\hline & Dependency & Relationships of the technique with others. \\
\hline \multirow[t]{8}{*}{ Cost } & Data quality level & $\begin{array}{l}\text { Requirements for identifying, preparing, and applying test } \\
\text { data, e.g., low, medium, high. }\end{array}$ \\
\hline & Formality level & $\begin{array}{l}\text { Level of using formalized structure and process, formal } \\
\text { logic, and mathematics in the technique, e.g., low, medium, } \\
\text { high. }\end{array}$ \\
\hline & Comprehensibility & $\begin{array}{l}\text { Effort required for understanding the technique, e.g., low, } \\
\text { medium, high. }\end{array}$ \\
\hline & Human resource & $\begin{array}{l}\text { Effort and time exposure required for applying the tech- } \\
\text { nique, e.g., low, medium, high. }\end{array}$ \\
\hline & Type of application & $\begin{array}{l}\text { How is the technique applied, coordinated teamwork or } \\
\text { single-handed? }\end{array}$ \\
\hline & Participant & Role(s) involved in application of the technique. \\
\hline & Knowledge & Knowledge required for applying the technique. \\
\hline & Experience & Experience required for applying the technique. \\
\hline
\end{tabular}


On the other hand, the category Cost includes the information related to the effort and time exposure required for understanding and mastering a $\mathrm{V} \& \mathrm{~V}$ technique, preparing testing data, and executing testing processes.

Applicability and Cost are similar to the two sides of one coin. The selection of the V\&V technique with both the best effectiveness and the lowest costs is hardly possible in practice. For example, compared with subjective techniques, a certain objective technique, may appear more effective, but its application is typically associated with high costs because of the high complexity level. Therefore when selecting V\&V techniques, the two types of technique characteristics should be analyzed and evaluated under consideration of the specified project environment, and consequently a reasonable balance between cost and benefit should be achieved. Table 1 describes the defined attributes in more detail.

\section{V\&V TECHNIQUES CATALOG}

Since the V\&V techniques summarized by Balci (1998b) can be regarded as (quasi-)complete from the practical point of view, Balci's taxonomy is used as a basis for establishing an organization-specific V\&V techniques catalog. As mentioned above, the $\mathrm{V} \& \mathrm{~V}$ techniques are categorized into four groups: informal, static, dynamic, and formal.

The category informal includes techniques like inspections and reviews, which heavily relay on subjective human decision-making. Static techniques are used to estimate the model design and implementation artifacts without model execution, such as structural analysis, interface analysis, and syntax analysis. Dynamic techniques, on the contrary, require model execution and evaluate the model based on its execution behavior. This category includes common testing techniques, e.g., white-box testing, black-box testing, debugging. Formal techniques are based on formal reasoning process and inference according to well-defined proof rules of the utilized specification language (MacKenzie, Schulmeyer, and Yilmaz 2002). Typical techniques in this category are induction, lambda calculus, proof of correctness, etc. Obviously, the level of mathematical formality required by a technique in each category increases from informal to formal and in general a high formality level also implies high complexity of a $V \& V$ technique.

In addition, since there exist a variety of $M \& S$ development and $V \& V$ processes with different definitions of phases, work products, roles, model deficiencies, and documentation requirements, the values of the scheme attributes $V \& V$ phase, Object, and Deficiency type are dependent on the life cycle process used in an M\&S project. In the context of this paper, a structured V\&V process - proposed by Brade (2000) and then extended by Wang and Lehmann (2007) - is considered. Regarding the determination of model deficiency types, Wang (2011) introduces a general classification framework, which can be used to classify model deficiencies for any structured $M \& S$ and $V \& V$ life cycle process.

For the purpose of 1.) evaluating the scheme feasibility and 2.) establishing a reliable V\&V catalog, two quality criteria are crucial and need to be considered when characterizing the V\&V techniques:

- Completeness: the proposed scheme should be able to characterize a given V\&V technique completely, i.e., each attribute is assigned an appropriate value;

- Uniqueness: the characteristics of a technique must be distinct, i.e., no two different techniques have an identical characterization.

The criterion completeness is determined by the ability of the scheme to capture the required information included in the individual V\&V techniques. Due to the lack of detailed knowledge about certain statistical and formal $V \& V$ techniques, some attributes values cannot be determined yet. Therefore, the default value unknown is temporarily set for the attributes concerned when characterizing techniques in the two families. The criterion uniqueness is principally determined by the ability to distinguish the different but similar V\&V techniques. Considering the descriptions in IEEE (1997), Balci (1998a), Gilb and Graham (1993), Schulmeyer and Mackenzie (2000), Figure 3 shows the comparison of three similar techniques inspections, reviews, and walkthroughs in the informal category. 


\begin{tabular}{|c|c|c|c|c|}
\hline Category & Attribute & Reviews & Inspections & Walkthroughs \\
\hline \multirow{11}{*}{ Applicability } & V\&V activity & Any & Any & Any \\
\hline & Object & $\begin{array}{l}\text { Project plans and } \\
\text { reports; } \\
\text { any model-related } \\
\text { work product and } \\
\text { documentation }\end{array}$ & $\begin{array}{l}\text { Any model-related } \\
\text { work product and } \\
\text { documentation }\end{array}$ & $\begin{array}{l}\text { Any model-related } \\
\text { work product and } \\
\text { documentation }\end{array}$ \\
\hline & Deficiency type & $\begin{array}{l}\text { Primary: } \\
\text { deficiencies in } \\
\text { project plans, } \\
\text { schedule and } \\
\text { management; } \\
\text { Secondary: } \\
\text { any M\&S } \\
\text { deficiency type }\end{array}$ & $\begin{array}{l}\text { Any M\&S } \\
\text { deficiency type }\end{array}$ & $\begin{array}{l}\text { Any M\&S } \\
\text { deficiency type }\end{array}$ \\
\hline & Development paradigm & Any & Any & Any \\
\hline & Modeling formalism & Any & Any & Any \\
\hline & Simulation type & Any & Any & Any \\
\hline & Simulation language & Any & Any & Any \\
\hline & Observable system & Not necessary & Not necessary & Not necessary \\
\hline & Model execution & Not necessary & Notnecessary & Not necessary \\
\hline & Modality & Subjective & Subjective & Subjective \\
\hline & Dependency & None & None & None \\
\hline \multirow{8}{*}{ Cost } & Data quality level & Low & Low to medium & Low \\
\hline & Formality level & Very low to low & $\begin{array}{l}\text { Low to medium } \\
\text { (5-phase process) }\end{array}$ & Low \\
\hline & Comprehensibility & High & High & High \\
\hline & Human resource & Medium to high & High & Medium \\
\hline & Type of application & Teamwork & Teamwork & Teamwork \\
\hline & Participant & $\begin{array}{l}\text { Decision maker, } \\
\text { review leader, } \\
\text { management staff, } \\
\text { technical staff, } \\
\text { and recorder }\end{array}$ & $\begin{array}{l}\text { Moderator, reader, } \\
\text { author, inspector, } \\
\text { and recorder }\end{array}$ & $\begin{array}{l}\text { Coordinator, } \\
\text { presenter (author), } \\
\text { team member } \\
\text { (development), } \\
\text { and recorder }\end{array}$ \\
\hline & Knowledge & None & None & None \\
\hline & Experience & None & $\begin{array}{l}\text { Training in } \\
\text { inspection } \\
\text { techniques }\end{array}$ & None \\
\hline
\end{tabular}

Figure 3: Characterization of the techniques reviews, inspections and walkthroughs 


\section{V\&V TECHNIQUES SELECTION STRATEGY}

The proposed selection strategy is based on the analysis of the techniques characteristics instantiated in the $\mathrm{V} \& \mathrm{~V}$ catalog compared with the actual project conditions, and can be integrated in an existing multistage tailoring process (Wang, Lehmann, and Karagkasidis 2009).

As shown in Figure 4, this tailoring process enables the adaptation of an M\&S life cycle process to project goals and environments, which are first characterized by a list of predefined project characteristics and then specified in the form of an application profile. Based on the application profile, the project-specific selection of essential work products, documentation, and activities for M\&S development and V\&V can be made respectively on four different levels (process level, product level, subject level, and role level). The proposed adaptation is conducted in two different ways: static and dynamic tailoring.

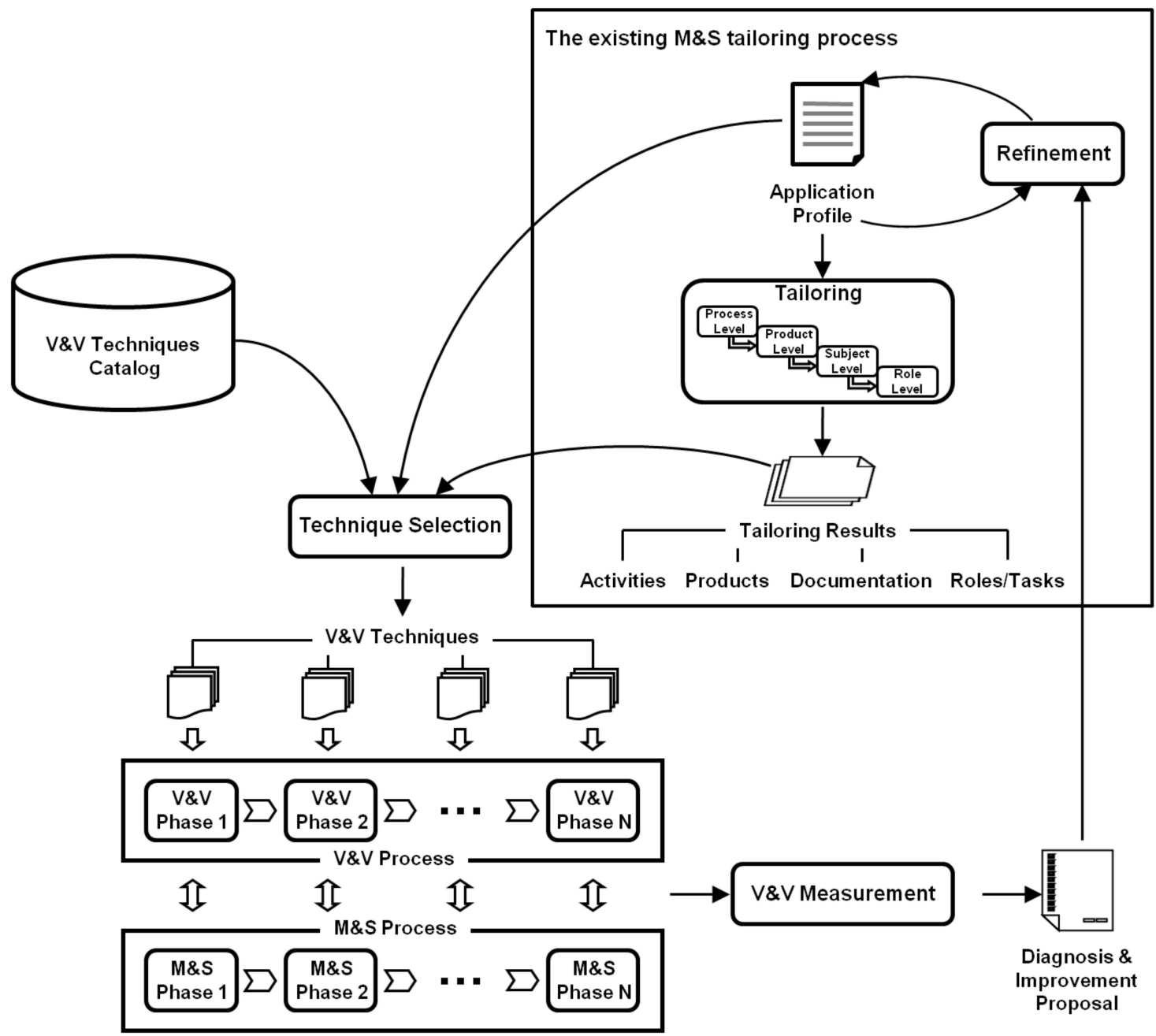

Figure 4: Extended planning and tailoring strategy

Static tailoring refers to selecting an initial set of project tasks based on the identified requirements and constraints at the beginning of an $M \& S$ project. The results of static tailoring are used primarily for the project management to develop the project management plan and quality assurance plan (including the $\mathrm{V} \& \mathrm{~V}$ plan). In the course of developing an M\&S application, however, new project characteristics could be continuously identified and even the determined requirements and constraints could be changed. Thus, the application profile should be accordantly refined and modified. Consequently a reselection of 
the determined project tasks should be made according to the current status of the application profile. This kind of adaptation is referred to as dynamic tailoring.

The selection of V\&V techniques can be applied as a conceptual extension of the existing adaptation process for both static and dynamic tailoring. For this purpose, several specific project characteristics relevant for techniques selection, such as simulation type, modeling formalism, development paradigm, simulation language, system observability, data quality required, technique objectivity required, human resource required, personnel qualification required, $\mathrm{V} \& \mathrm{~V}$ level required, etc., are additionally specified and integrated in the application profile.

Thus, the technique selection for each $V \& V$ phase is conducted by evaluating the available techniques included in the V\&V catalog in comparison with the actual project conditions, which are specified as characteristics values in the application profile and as a list of tailoring results. If the selection provides more than one techniques for a certain $\mathrm{V} \& \mathrm{~V}$ phase, either one or a combination of them could be used. If, however, no suitable technique can be selected, i.e., in the current V\&V catalog there exits no technique exactly matching the specified project characteristics, two attempts should be followed prior to reselection: 1.) relaxing one or more values of the project characteristics in the application profile such as requirements of data quality level or personnel qualification; or/and 2.) extending the $\mathrm{V} \& \mathrm{~V}$ catalog with additional techniques. In the context of static tailoring, the selected $V \& V$ techniques for each $V \& V$ phase are also essential for completing the project management plan and quality assurance plan and should be documented in the V\&V plan.

Associated with the technique selection process, a V\&V measurement tool (Wang 2011) can be used to analyze and evaluate the results of the $\mathrm{V} \& \mathrm{~V}$ effort, e.g., effectiveness of selected $\mathrm{V} \& \mathrm{~V}$ techniques, propagation of $M \& S$ deficiencies, impact of a techniques selection on a certain $V \& V$ activity, etc. This measurement approach proposes a model deficiency classification based on the observation that M\&S deficiencies, despite their negative impact on the assessment issue, carry a large amount of insightful information about the $\mathrm{V} \& \mathrm{~V}$ process, such as where in the model documentation, in which $\mathrm{V} \& \mathrm{~V}$ activity (when), and by which $\mathrm{V} \& \mathrm{~V}$ technique (how), an $\mathrm{M} \& \mathrm{~S}$ deficiency was detected. The advantage of using this approach is that it measures model $V \& V$ being conducted by means of quantitative evidences and is able to provide a process diagnosis for identifying improvement potentials. The feedback of $\mathrm{V} \& \mathrm{~V}$ measurement may indicate problem areas of the model development and V\&V activities conducted so far, needs of rework or application of more effective V\&V techniques, etc. Consequently, the actual project characteristics should be updated, and dynamic tailoring could be therefore triggered for selecting necessary project tasks as well as $\mathrm{V} \& \mathrm{~V}$ techniques.

\section{CONCLUSION}

Effective selection of appropriate $\mathrm{V} \& \mathrm{~V}$ techniques is an essential requirement for conducting model $\mathrm{V} \& \mathrm{~V}$. When selecting $\mathrm{V} \& \mathrm{~V}$ techniques, a major concern is how to identify the relevant information necessary for decision-making. The key to solve this problem is 1.) to investigate the characteristics of the available $\mathrm{V} \& \mathrm{~V}$ techniques and 2.) to determine constraints of their application.

This paper introduces a characterization approach to establishing a $\mathrm{V} \& \mathrm{~V}$ techniques catalog which packages the characterized techniques together with the information about their applicability and the costs required. The defined scheme has an open structure, new attributes for additional characterization aspects can be included. For example, a new attribute Supporting tool, which refers to the facilitation of applying a $\mathrm{V} \& \mathrm{~V}$ technique, is conceivable.

In addition, a techniques selection strategy is proposed, which is then integrated in the existing M\&S tailoring process. Thus, the extended planning and tailoring concept consists of four components: 1.) project-specific selection of activities, products, and documentation; 2.) project-specific selection of $V \& V$ techniques; 3.) measurement of $\mathrm{V} \& \mathrm{~V}$ being conducted; and 4.) refinement of project characteristics for dynamic tailoring. 
Since it is situation dependent to determine which $V \& V$ technique is appropriate for a particular V\&V activity, rigorous and precise specification of $M \& S$ objectives and requirements is of crucial importance for the proposed characterization approach. As discussed in (Tolk 2012), the need of using formal methods to capture and specify the intended purpose of an $M \& S$ application is obvious. On the other hand, for the purpose of providing rapid and flexible response to ever-changing requirements, agile methodologies are introduced in the M\&S development process, e.g., the DEVS Unified Process (Mittal and Martín 2013). As already mentioned, the proposed techniques characterization, due to its generic nature, is applicable to any structured model development and $\mathrm{V} \& \mathrm{~V}$ precess. The extent to which this approach can be used for agile development paradigm depends, however, on whether a structured M\&S process with agile characteristics or an agile process without structure is deployed. Investigating the impact of the agile practices on application and selection of $\mathrm{V} \& \mathrm{~V}$ techniques is one of our future research goals.

\section{REFERENCES}

Arthur, J. D., and R. E. Nance. 2000. "Verification and Validation without Independence: A Recipe for Failure". In Proceedings of the 2000 Winter Simulation Conference, edited by J. Joines, R. Barton, K. Kang, and P. Fishwick, 859-865.

Balci, O. 1994. "Validation, Verification, and Testing Techniques Throughout the Life Cycle of a Simulation Study". Annals of Operations Research 53 (1): 121-173.

Balci, O. 1998a. "Verification, Validation and Accreditation". In Proceedings of the 1998 Winter Simulation Conference, edited by D. Medeiros, E. Watson, J. Carson, and M. Manivannan, 41-48.

Balci, O. 1998b. "Verification, Validation, and Testing". In Handbook of Simulation, edited by J. Banks, Chapter 10, 335-393. John Wiley \& Sons.

Balci, O., R. E. Nance, J. D. Arthur, and W. F. Ormsby. 2002. "Expanding our Horizons in Verification, Validation, and Accreditation Research and Practice". In Proceedings of the 2002 Winter Simulation Conference, edited by E. Yücesan, C.-H. Chen, J. Snowdon, and J. Charnes, 653-663.

Brade, D. 2000. "Enhancing Modeling and Simulation Accreditation by Structuring Verification and Validation Results". In Proceedings of the 2000 Winter Simulation Conference, edited by J. Joines, R. Barton, K. Kang, and P. Fishwick.

Department of Defense 2001. VV\&A Recommended Practices Guide (RPG) for $V \& V$ Techniques (version 8/15/01). Department of Defense. Available via http://vva.msco.mil/Ref_Docs/VVTechniques/VVtechniques-pr.PDF [last accessed August 2013].

Deslandres, V., and H. Pierreval. 1991. "An Expert System Prototype Assisting the Statistical Validation of Simulation Models". Simulation 56 (2): 79-89.

Dias-Neto, A. C., and C. H. Travassos. 2009. "Evaluation of \{model-based Testing Techniques Selection Approaches: An External Replication". In Proceedings of the Third International Symposium on Empirical Software Engineering and Measurement, 269-278: IEEE.

Gilb, T., and D. Graham. 1993. Software Inspection. Addison-Wesley.

IEEE 1990. IEEE Standard Glossary of Software Engineering Terminology. IEEE std 610.12-1990.

IEEE 1997. IEEE Standard for Software Reviews. IEEE std 1028-1997.

Law, A. M., and W. D. Kelton. 2007. Simulation Modeling and Analysis. Fourth ed. McGraw-Hill.

MacKenzie, G. R., G. G. Schulmeyer, and L. Yilmaz. October 22-24, 2002. "Verification Technology Potential with Different Modeling and Simulation Development and Implementation Paradigms". In Proceedings of Foundations for V\&V in the 21st Century Workshop. Laurel, Maryland, USA.

Mittal, S., and J. L. R. Martín. 2013. "DEVS Unified Process". In Netcentric System of Systems Engineering with DEVS Unified Process, Chapter 6, 169-188. CRC Press.

Oberkampf, W. L., S. M. DeLand, B. M. Rutherford, K. V. Diegert, and K. F. Alvin. 2002. "Error and uncertainty in modeling and simulation". Reliability Engineering and System Safety 75 (3): 333-357. 
Overstreet, C. M. 2002. "Model Testing: Is it only a Special Case of Software Testing". In Proceedings of the 2002 Winter Simulation Conference, edited by E. Yücesan, C.-H. Chen, J. Snowdon, and J. Charnes, 641-647.

Sargent, R. G. 2005. "Verification and Validation of Simulation Models". In Proceedings of the 2005 Winter Simulation Conference, edited by M. E. Kuhl, N. M. Steiger, F. B. Armstrong, and J. A. Joines, 130-143.

Schulmeyer, G. G., and G. R. Mackenzie. 2000. Verification and Validation of Modern Software-Intensive Systems. Upper Saddle River, NJ: Prentice Hall.

Shannon, R. E. 1975. Systems Simulation - the Art and Science. Englewood Cliffs, N.J.: Prentice-Hall.

Shannon, R. E. 1998. "Introduction to the Art and Science of Simulation". In Proceedings of the 1998 Winter Simulation Conference, edited by D. Medeiros, E. Watson, J. Carson, and M. Manivannan, 7-14.

Tolk, A. 2012. "Verification and Validation". In Engineering Principles of Combat Modeling and Distributed Simulation, edited by A. Tolk, Chapter 14, 263-294. John Wiley \& Sons.

Vegas, S. 2002. Characterization Schema for Selecting Software Testing Techniques. Ph. D. thesis, Facultad de Informática. Universidad Politécnica de Madrid.

Vegas, S., and V. Basili. 2005. "A Characterization Schema for Software Testing Techniques". Empirical Software Engineering 10 (4): 437-466.

Vegas, S., N. Juristo, and V. Basili. 2003. "A Process for Identifying Relevant Information for a Repository: A Case Study for Testing Techniques”. In Managing Software Engineering Konowledge, Chapter 10, 190-230. Berlin/Heidelberg: Springer-Verlag.

Walton, G. H., R. M. Patton, and D. J. Parsons. 2001. "Usage Testing of Military Simulation Systems". In Proceedings of the 2001 Winter Simulation Conference, edited by B. Peters, J. Smith, D. Medeiros, and M. Rohrer, 771-779.

Wang, Z. 2011. "Towards a Measurement Tool for Verification and Validation of Simulation Models". In Proceedings of the 2011 Winter Simulation Conference, edited by S. Jain, R. Creasey, J. Himmelspach, K. White, and M. Fu.

Wang, Z., and A. Lehmann. 2007. "Verification and Validation of Simulation Models and Applications: A Methodological Approach". In Recent Advances in Modeling and Simulation Tools for Communication Networks and Services, edited by A. N. Ince and A. Bragg, Chapter 11, 227-240. New York: Springer.

Wang, Z., and A. Lehmann. 2010. "Quality Assurance of Models and Simulation Applications". International Journal of Modeling, Simulation, and Scientific Computing 1 (1): 27-45.

Wang, Z., A. Lehmann, and A. Karagkasidis. 2009. "A Multistage Approach for Quality- and EfficiencyRelated Tailoring of Modelling and Simulation Processes". Simulation News Europe 19 (2): 12-20.

Wojcicki, M. A., and P. Strooper. 2007. "An Iterative Empirical Strategy for the Systematic Selection of a Combination of Verification and Validation Technologies". In Proceedings of the Fifth International Workshop on Software Quality (WoSQ 2007). Washington, DC, USA.

Zeigler, B. P., H. Praehofer, and T. G. Kim. 2000. Theory of Modeling and Simulation. Second ed. Academic Press.

\section{AUTHOR BIOGRAPHIES}

Zhongshi Wang is a Research Associate at ITIS GmbH at the University of the Federal Armed Forces in Munich, Germany. His research interests include verification, validation, and accreditation (VV\&A) of simulation models, model documentation, and tailoring of modeling and simulation processes. His email and web addresses are zhongshi.wang@unibw.de and http://www.unibw.de/zhongshi.wang. 